\title{
Stopping a Viscous Fluid by a Feedback Dissipative Field: Thermal Effects without Phase Changing
}

\author{
S.N. Antontsev, J.I. Díaz and H.B. de Oliveira
}

Dedicated to Professor V.A. Solonnikov on the occasion of his 70th birthday.

\begin{abstract}
We show how the action on two simultaneous effects (a suitable coupling about velocity and temperature and a low range of temperature but upper that the phase changing one) may be responsible of stopping a viscous fluid without any changing phase. Our model involves a system, on an unbounded pipe, given by the planar stationary Navier-Stokes equation perturbed with a sublinear term $\mathbf{f}(\mathbf{x}, \theta, \mathbf{u})$ coupled with a stationary (and possibly nonlinear) advection diffusion equation for the temperature $\theta$.

After proving some results on the existence and uniqueness of weak solutions we apply an energy method to show that the velocity $\mathbf{u}$ vanishes for $\mathbf{x}$ large enough.
\end{abstract}

Mathematics Subject Classification (2000). 76A05 ,76D07, 76E30, 35G15.

Keywords. Non-Newtonian fluids, nonlinear thermal diffusion equations, feedback dissipative field, energy method, heat and mass transfer, localization effect.

\section{Introduction}

It is well known (see, for instance, $[6,8,14]$ ) that in phase changing flows (as the Stefan problem) usually the solid region is assumed to remain static and so we can understand the final situation in the following way: the thermal effect are able to stop a viscous fluid.

The main contribution of this paper is to show how the action on two simultaneous effects (a suitable coupling about velocity and temperature and a low range of temperature but upper the phase changing one) may be responsible of stopping a viscous fluid without any changing phase. This philosophy could be useful in the monitoring of many flows problems, specially in metallurgy. 
We shall consider a, non-standard, Boussinesq coupling among the temperature $\theta$ and the velocity $\mathbf{u}$. Motivated by our previous works (see $[1,2,3,4]$ ), we assume the body force field is given in a non-linear feedback form, $\mathbf{f}: \Omega \times \mathbb{R} \times \mathbb{R}^{2} \rightarrow$ $\mathbb{R}^{2}, \mathbf{f}=\left(f_{1}(\mathbf{x}, \theta, \mathbf{u}), f_{2}(\mathbf{x}, \theta, \mathbf{u})\right)$, where $\mathbf{f}$ is a Carathéodory function (i.e., continuous on $\theta$ and $\mathbf{u}$ and measurable in $\mathbf{x})$ such that, for every $\mathbf{u} \in \mathbb{R}^{2}, \mathbf{u}=(u, v)$, for any $\theta \in[m, M]$, and for almost all $\mathbf{x} \in \Omega$

$$
-\mathbf{f}(\mathbf{x}, \theta, \mathbf{u}) \cdot \mathbf{u} \geq \delta \chi_{\mathbf{f}}(\mathbf{x})|u|^{1+\sigma(\theta)}-g(\mathbf{x}, \theta)
$$

for some $\delta>0, \sigma$ a Lipschitz continuous function such that

$$
0<\sigma^{-} \leq \sigma(\theta) \leq \sigma^{+}<1, \quad \theta \in[m, M],
$$

and

$$
g \in \mathrm{L}^{1}\left(\Omega^{x_{g}} \times \mathbb{R}\right), \quad g \geq 0, g(\mathbf{x}, \theta)=0 \text { a.e. in } \Omega^{x_{g}} \text { for any } \theta \in[m, M],
$$

for some $x_{\mathbf{f}}, x_{g}$, with $0 \leq x_{g}<x_{\mathbf{f}} \leq \infty$ and $x_{\mathbf{f}}$ large enough, where $\Omega^{x_{g}}=\left(0, x_{g}\right) \times$ $(0, L)$ and $\Omega_{x_{g}}=\left(x_{g}, \infty\right) \times(0, L)$. The function $\chi_{\mathbf{f}}$ denotes the characteristic function of the interval $\left(0, x_{\mathbf{f}}\right)$, i.e., $\chi_{\mathbf{f}}(\mathbf{x})=1$, if $x \in\left(0, x_{\mathbf{f}}\right)$ and $\chi_{\mathbf{f}}(\mathbf{x})=0$, if $x \notin\left(0, x_{\mathbf{f}}\right)$. We shall not need any monotone dependence assumption on the function $\sigma(\theta)$.

It seems interesting to notice that the term $\mathbf{f}(\mathbf{x}, \theta, \mathbf{u})$ plays a similar role to the one in the penalized changing phase problems (see equation (3.13) of [14]), although our formulation and our methods of proof are entirely different. We shall prove that the fluid is stopped at a finite distance of the semi-infinite strip entrance by reducing the nonlinear system to a fourth order non-linear scalar equation for which the localization of solutions is obtained by means of a suitable energy method (see [5]).

\section{Statement of the problem}

In the domain $\Omega=(0, \infty) \times(0, L), L>0$, we consider a planar stationary thermal flow of a fluid governed by the following system

$$
\begin{gathered}
(\mathbf{u} \cdot \nabla) \mathbf{u}=\nu \triangle \mathbf{u}-\nabla p+\mathbf{f}(\mathbf{x}, \theta, \mathbf{u}), \\
\operatorname{div} \mathbf{u}=0, \\
\mathbf{u} \cdot \nabla \mathcal{C}(\theta)=\Delta \varphi(\theta),
\end{gathered}
$$

where $\mathbf{u}=(u, v)$ is the vector velocity of the fluid, $\theta$ its absolute temperature, $p$ is the hydrostatic pressure, $\nu$ is the kinematics viscosity coefficient,

$$
\mathcal{C}(\theta):=\int_{\theta_{0}}^{\theta} C(s) d s \quad \text { and } \quad \varphi(\theta):=\int_{\theta_{0}}^{\theta} \kappa(s) d s,
$$

with $C(\theta)$ and $\kappa(\theta)$ being the specific heat and the conductivity, respectively. Assuming $\kappa>0$ then $\varphi$ is invertible and so $\theta=\varphi^{-1}(\bar{\theta})$ for some real argument $\bar{\theta}$. Then we can define functions

$$
\overline{\mathcal{C}}(\bar{\theta}):=\mathcal{C} \circ \varphi^{-1}(\bar{\theta}), \quad \overline{\mathbf{f}}(\mathbf{x}, \bar{\theta}, \mathbf{u}):=\mathbf{f} \circ \varphi^{-1}(\bar{\theta}), \quad \bar{\mu}(\bar{\theta}):=\mu \circ \varphi^{-1}(\bar{\theta}) .
$$


We point out that functions $\overline{\mathcal{C}}, \overline{\mathbf{f}}$ and $\bar{\mu}$ are Lipschitz continuous functions of $\bar{\theta}$. Substituting these expressions in (2.4)-(2.6), we get, omitting the bars,

$$
\begin{gathered}
(\mathbf{u} \cdot \nabla) \mathbf{u}=\nu \Delta \mathbf{u}-\nabla p+\mathbf{f}(\mathbf{x}, \theta, \mathbf{u}), \\
\operatorname{div} \mathbf{u}=0, \\
\mathbf{u} \cdot \nabla \mathcal{C}(\theta)=\triangle \theta .
\end{gathered}
$$

To these equations we add the following boundary conditions on $\mathbf{u}$

$$
\begin{gathered}
\mathbf{u}=\mathbf{u}_{*}, \quad \text { on } x=0, \\
\mathbf{u}=\mathbf{0}, \quad \text { on } y=0, L, \\
\mathbf{u} \rightarrow \mathbf{0}, \quad \text { when } x \rightarrow \infty,
\end{gathered}
$$

and on $\theta$

$$
\begin{gathered}
\theta=\theta_{*}, \quad \text { on } x=0, y=0, L, \\
\theta \rightarrow 0, \quad \text { when } x \rightarrow \infty,
\end{gathered}
$$

where $\mathbf{u}_{*}$ and $\theta_{*}$ are given functions with a suitable regularity to be indicated later on and

$$
0 \leq m \leq \theta_{*}(\mathbf{x}) \leq M<\infty .
$$

We assume the possible non-zero velocity $\mathbf{u}_{*}$ and temperature $\theta_{*}$ satisfy the compatibility conditions

$$
\begin{gathered}
\mathbf{u}_{*}(0)=\mathbf{u}_{*}(L)=\mathbf{0}, \quad \int_{0}^{L} u_{*}(s) d s=0, \\
\theta_{*}(x, y) \rightarrow 0, \quad \text { when } x \rightarrow \infty \text { for any } y \in[0, L] .
\end{gathered}
$$

\section{Existence theorem}

As in $[1,2,3,4]$, we introduce the functional spaces

$$
\begin{gathered}
\widetilde{\mathbf{H}}(\Omega)=\left\{\mathbf{u} \in \mathbf{H}(\Omega): \mathbf{u}(0, .)=\mathbf{u}_{*}(.), \mathbf{u}(., 0)=\mathbf{u}(., L)=\mathbf{0}, \lim _{x \rightarrow \infty}|\mathbf{u}|=0\right\}, \\
\widetilde{\mathbf{H}}_{0}(\Omega)=\left\{\mathbf{u} \in \mathbf{H}(\Omega): \mathbf{u}(0, .)=\mathbf{u}(., 0)=\mathbf{u}(., L)=\mathbf{0}, \lim _{x \rightarrow \infty}|\mathbf{u}|=0\right\},
\end{gathered}
$$

where $\mathbf{H}(\Omega)=\left\{\mathbf{u} \in \mathbf{H}^{1}(\Omega): \operatorname{div} \mathbf{u}=0\right\}$, and assume that

$$
\mathbf{u}_{*} \in \mathbf{H}^{\frac{1}{2}}(0, L) .
$$

We shall search solutions $(\theta, \mathbf{u})$ such that, additionally to assumptions (2.14) and (2.12), satisfy

$$
\int_{\Omega}|\nabla \theta|^{2} d \mathbf{x}<\infty \quad \text { and } \quad \int_{\Omega}|\nabla \mathbf{u}|^{2} d \mathbf{x}<\infty .
$$

Moreover, due to the fact that the Poincaré inequality

$$
\int_{\Omega}|w|^{p} d \mathbf{x} \leq\left(\frac{L}{\pi}\right)^{p} \int_{\Omega}|\nabla w|^{p} d \mathbf{x}
$$


holds for every $w \in \mathrm{W}_{0}^{1, p}(\Omega)$ and $1 \leq p<\infty$ (see, e.g., [10]), our searched solutions $(\theta, \mathbf{u})$ will be elements of the Sobolev space $\mathrm{H}^{1}(\Omega) \times \mathbf{H}^{1}(\Omega)$.

Let us still denote by $\mathbf{u}_{*}$ and $\theta_{*}$ the extensions of the boundary data to the whole domain $\Omega$ in a way such that

$$
\mathbf{u}_{*} \in \widetilde{\mathbf{H}}(\Omega) \quad \text { and } \quad \theta_{*} \in \mathrm{W}^{1, q}(\Omega) \cap \mathrm{C}^{\alpha}(\bar{\Omega}), \quad 2<q<\infty, \alpha>0 .
$$

Definition 3.1. The pair $(\theta, \mathbf{u})$ is said to be a weak solution of (2.7)-(2.14) if:

(i) $\theta-\theta_{*} \in \mathrm{W}_{0}^{1, q}(\Omega) \cap \mathrm{C}^{\alpha}(\bar{\Omega}), \alpha>0,2<q<\infty, m \leq \theta \leq M$ and for any test function $\zeta \in \mathrm{W}_{0}^{1, q^{\prime}}(\Omega)\left(1 / q+1 / q^{\prime}=1\right)$

$$
\int_{\Omega}(\nabla \theta-\mathcal{C}(\theta) \mathbf{u}) \cdot \nabla \zeta d \mathbf{x}=0 .
$$

(ii) $\mathbf{u} \in \widetilde{\mathbf{H}}(\Omega), \mathbf{u}-\mathbf{u}_{*} \in \widetilde{\mathbf{H}}_{0}(\Omega), \mathbf{f}(\mathbf{x}, \theta(\mathbf{x}), \mathbf{u}(x)) \in \mathbf{L}_{\text {loc }}^{1}(\Omega)$ and for every $\varphi \in$ $\widetilde{\mathbf{H}}_{0}(\Omega) \cap \mathbf{L}^{\infty}(\Omega)$ with compact support,

$$
\nu \int_{\Omega} \nabla \mathbf{u}: \nabla \varphi d \mathbf{x}+\int_{\Omega} \mathbf{u} \cdot \nabla \mathbf{u} \cdot \varphi d \mathbf{x}=\int_{\Omega} \mathbf{f} \cdot \varphi d \mathbf{x} .
$$

In this section, we shall assume that $\mathbf{f}: \Omega \times \mathbb{R} \times \mathbb{R}^{2} \rightarrow \mathbb{R}^{2}$ is given by the structural condition

$$
\mathbf{f}(\mathbf{x}, \theta, \mathbf{u})=-\delta \chi_{\mathbf{f}}(\mathbf{x})\left(|u|^{\sigma(\theta)-1} u, 0\right)-\mathbf{h}(\mathbf{x}, \theta, \mathbf{u}),
$$

for any $\mathbf{u}=(u, v)$, any $\theta \in[m, M]$ and almost every $\mathbf{x} \in \Omega$, for some $\delta>0$, $0 \leq x_{\mathbf{f}} \leq \infty$ and $\sigma(\theta)$ satisfies (1.2). Here, $\mathbf{h}(\mathbf{x}, \theta, \mathbf{u})$ is a Carathéodory function such that

$$
\mathbf{h}(\mathbf{x}, \theta, \mathbf{u}) \cdot \mathbf{u} \geq-g(\mathbf{x}, \theta)
$$

for every $\mathbf{u} \in \mathbb{R}^{2}$, for any $\theta \in[m, M]$ and almost all $\mathbf{x} \in \Omega$, for some function $g$ satisfying (1.3), and we assume

$$
H_{K} \in \mathrm{L}^{1}\left(\Omega^{x_{\mathbf{f}}}\right) \text { for all } K>0, \quad H_{K}(\mathbf{x})=\sup _{|\mathbf{u}| \leq K, \theta \in[m, M]}|\mathbf{h}(\mathbf{x}, \theta, \mathbf{u})| .
$$

Theorem 3.1. Under conditions (1.2), (2.15)-(2.17), (3.18), (3.20) and (3.22)(3.24), the problem (2.7)-(2.14) has, at least, one weak solution $(\theta, \mathbf{u})$.

Proof. We will prove this theorem in several steps.

First step: an auxiliary problem for the temperature $\theta$. Let

$$
\mathbf{w} \in \mathbf{L}^{2}(\Omega) \cap \mathbf{L}^{q}(\Omega), \quad \text { with } 2<q<\infty,
$$

be a given function and let us consider the following problem for the temperature

$$
\mathbf{w} \cdot \nabla \mathcal{C}(\theta)=\triangle \theta
$$

completed with the boundary conditions (2.13)-(2.14). Since $\mathcal{C}$ is Lipschitz continuous we know (see, e.g., $[7,10,11])$ that problem $(3.26),(2.13)-(2.14)$, assuming (3.25), has a unique weak solution $\theta$ such that

$$
\|\theta\|_{\mathrm{W}^{1, q}(\Omega)},\|\theta\|_{\mathrm{C}^{\alpha}(\bar{\Omega})} \leq C\left(q,\|\mathbf{w}\|_{\mathbf{L}^{q}(\Omega)},\left\|\theta_{*}\right\|_{\mathrm{W}^{1, q}(\Omega)}\right)
$$


where $\alpha=1+[2 / q]-2 / q>0$. Moreover, from the Maximum Principle,

$$
m \leq \theta(x) \leq M \text {. }
$$

Then we can define the non-linear operator

$$
\Lambda: \mathbf{L}^{2}(\Omega) \cap \mathbf{L}^{q}(\Omega) \rightarrow \mathrm{W}^{1, q}(\Omega) \cap \mathrm{C}^{\alpha}(\bar{\Omega}), \quad \Lambda(\mathbf{w})=\theta,
$$

with $\alpha=1+[2 / q]-2 / q>0,2<q<\infty$. The operator $\Lambda$ is continuous, because from $[10,11]$, we get that given a sequence $\mathbf{w}_{n}$ such that

$$
\left\|\mathbf{w}_{n}-\mathbf{w}\right\|_{\mathbf{L}^{2}(\Omega)}+\left\|\mathbf{w}_{n}-\mathbf{w}\right\|_{\mathbf{L}^{q}(\Omega)} \rightarrow 0, \quad \text { as } n \rightarrow \infty,
$$

then

$$
\left\|\Lambda\left(\mathbf{w}_{n}\right)-\Lambda(\mathbf{w})\right\|_{\mathrm{W}^{1, q}(\Omega)}+\left\|\Lambda\left(\mathbf{w}_{n}\right)-\Lambda(\mathbf{w})\right\|_{\mathrm{C}^{\alpha}(\bar{\Omega})} \rightarrow 0, \quad \text { as } n \rightarrow \infty .
$$

Second step: an auxiliary problem for the velocity $\mathbf{u}$. Let $\omega$ be a given function such that

$$
\omega \in \mathrm{W}^{1, q}(\Omega) \cap \mathrm{C}^{\alpha}(\bar{\Omega}), \quad 2<q<\infty, \quad \alpha>0, \quad m \leq \omega \leq M
$$

and let us consider the problem for the velocity constituted by the following equation of motion

$$
(\mathbf{u} \cdot \nabla) \mathbf{u}=\nu \triangle \mathbf{u}-\nabla p+\mathbf{f}(\mathbf{x}, \omega, \mathbf{u}),
$$

the equation of continuity (2.8) and the boundary conditions (2.10)-(2.12). Applying the results of [4] (which is possible due to the assumptions (3.22)-(3.24) and (3.29)), the problem (3.30), (2.8), (2.10)-(2.12) has, at least, one weak solution $\mathbf{u} \in \mathbf{H}^{1}(\Omega)$ which satisfies

$$
\int_{\Omega}\left(|\nabla \mathbf{u}|^{2}+\chi_{\mathbf{f}}|u|^{1+\sigma(\theta)}+|\mathbf{h}(\mathbf{x}, \omega, \mathbf{u}) \cdot \mathbf{u}|\right) d \mathbf{x} \leq C,
$$

where

and, in fact,

$$
C=C\left(L, m, M, \delta, \nu,\left\|\mathbf{u}_{*}\right\|_{\mathbf{H}^{\frac{1}{2}}(0, L)},\|g\|_{\mathrm{L}^{1}\left(\Omega^{x} \times \mathbb{R}\right)}\right)
$$

$$
C=C_{0}\left(L, m, M, \delta, \nu\left\|\mathbf{u}_{*}\right\|_{\mathbf{H}^{\frac{1}{2}(0, L)}}^{2}\right),
$$

if $g=0$. Then we can define the non-linear operator

$$
\Pi: \mathrm{W}^{1, q}(\Omega) \cap \mathrm{C}^{\alpha}(\bar{\Omega}) \rightarrow \mathbf{L}^{q}(\Omega), \quad \Pi(\omega)=\mathbf{u},
$$

with $2<q<\infty$ and $\alpha>0$, which is continuous.

Third step: application of Schauder's theorem. Given $q>2$, formulas (3.28) and (3.32) allow to define the composition non-linear operator

$$
\Upsilon=\Pi \Lambda: \mathbf{L}^{2}(\Omega) \cap \mathbf{L}^{q}(\Omega) \rightarrow \mathbf{L}^{q}(\Omega) .
$$

From (3.31) we get that $\Upsilon$ transforms $\mathbf{L}^{2}(\Omega) \cap \mathbf{L}^{q}(\Omega)$ into a bounded subset of $\mathbf{H}^{1}(\Omega)$ and, from the Sobolev compact embedding $\mathbf{H}^{1}(\Omega) \rightarrow \mathbf{L}^{q}(\Omega), 2<q<\infty$, it is completely continuous. Then, according to Schauder's theorem, (3.33) has, at 
least, a fixed point. This proves the existence of a weak solution $(\theta, \mathbf{u})$ to problem $(2.7)-(2.14)$.

Remark 3.1. Questions about the solvability of boundary value problems for the Navier-Stokes system in domains with noncompact boundaries were discussed by many authors amongst whom Solonnikov [13].

\section{Uniqueness of weak solution}

For the sake of simplicity in the exposition we will assume in this section that the coupling thermal force obeys to the special form

$$
\mathbf{f}(\mathbf{x}, \theta, \mathbf{u})=-\delta \chi_{\mathbf{f}}(\mathbf{x})\left(|u(\mathbf{x})|^{\sigma(\theta)-1} u(\mathbf{x}), 0\right) .
$$

The main result of this section, concerning the uniqueness of solutions, is the following.

Theorem 4.1. Assume (1.2) and (2.15)-(2.17). We additionally suppose that

$$
\left|\mathcal{C}^{\prime}(\theta)\right| \leq \lambda \quad \text { for every } \theta \in[m, M]
$$

and

$$
\left|\sigma^{\prime}(\theta)\right| \leq \lambda \quad \text { for every } \theta \in[m, M],
$$

for $\lambda \leq \lambda^{*}$ and for some small enough positive constant $\lambda^{*}>0$. Then, if $\left\|\mathbf{u}_{*}\right\|_{\mathbf{H}^{\frac{1}{2}}(0, L)} \leq \varepsilon^{*}$ for some small enough positive constant $\varepsilon^{*}>0$, the problem (2.7)-(2.14), (4.34) has a unique weak solution $(\mathbf{u}, \theta)$.

Proof. Let $\left(\theta_{1}, \mathbf{u}_{1}\right), \mathbf{u}_{1}=\left(u_{1}, v_{1}\right)$, and $\left(\theta_{2}, \mathbf{u}_{2}\right), \mathbf{u}_{2}=\left(u_{2}, v_{2}\right)$, be two weak solutions to problem (2.7)-(2.14) and let us set $\theta=\theta_{1}-\theta_{2}, \mathbf{u}=\mathbf{u}_{1}-\mathbf{u}_{2}$. According to Definition 3.1, $\mathbf{u}=\mathbf{u}_{\mathbf{1}}-\mathbf{u}_{\mathbf{2}} \in \mathbf{H}_{0}^{1}(\Omega)$ and $\theta=\theta_{1}-\theta_{2} \in \mathrm{H}_{0}^{1}(\Omega)$. Moreover, functions $\theta$, $\mathbf{u}$ satisfy to

$$
\begin{gathered}
\int_{\Omega}\left[\nabla \theta-\left(\mathcal{C}\left(\theta_{1}\right) \mathbf{u}_{1}-\mathcal{C}\left(\theta_{2}\right) \mathbf{u}_{2}\right) \mathbf{u}\right] \cdot \nabla \zeta d \mathbf{x}=0, \\
\nu \int_{\Omega} \nabla \mathbf{u}: \nabla \varphi d \mathbf{x}+\int_{\Omega}\left[\left(\mathbf{u}_{1} \cdot \nabla\right) \mathbf{u}_{1}-\left(\mathbf{u}_{2} \cdot \nabla\right) \mathbf{u}_{2}\right] \cdot \varphi d \mathbf{x} \\
=-\delta \int_{\Omega} \chi_{\mathbf{f}}(\mathbf{x})\left(\left|u_{1}(\mathbf{x})\right|^{\sigma\left(\theta_{1}\right)-1} u_{1}(\mathbf{x})-\left|u_{2}(\mathbf{x})\right|^{\sigma\left(\theta_{2}\right)-1} u_{2}(\mathbf{x}), 0\right) \cdot \varphi d \mathbf{x} .
\end{gathered}
$$

Setting $\zeta=\theta$ and $\varphi=\mathbf{u}$, we came to the relations

$$
\begin{gathered}
\int_{\Omega}|\nabla \theta|^{2} d \mathbf{x}=\int_{\Omega}\left(\mathcal{C}\left(\theta_{1}\right)-\mathcal{C}\left(\theta_{2}\right)\right) \mathbf{u}_{1} \cdot \nabla \theta d \mathbf{x} \\
+\int_{\Omega} \mathcal{C}\left(\theta_{2}\right) \mathbf{u} \cdot \nabla \theta d \mathbf{x}:=J_{1}+J_{2}, \\
\nu \int_{\Omega}|\nabla \mathbf{u}|^{2} d \mathbf{x}+I_{1}=I_{2}+I_{3},
\end{gathered}
$$


where

$$
\begin{gathered}
I_{1}:=\delta \int_{\Omega} \chi_{\mathbf{f}}(\mathbf{x})\left(\left|u_{1}(\mathbf{x})\right|^{\sigma\left(\theta_{1}\right)-1} u_{1}(\mathbf{x})-\left|u_{2}(\mathbf{x})\right|^{\sigma\left(\theta_{1}\right)-1} u_{2}(\mathbf{x}), 0\right) \cdot \mathbf{u} d \mathbf{x} \\
I_{2}:=-\delta \int_{\Omega} \chi_{\mathbf{f}}(\mathbf{x})\left(\left|u_{2}(\mathbf{x})\right|^{\sigma\left(\theta_{1}\right)-1} u_{2}(\mathbf{x})-\left|u_{2}(\mathbf{x})\right|^{\sigma\left(\theta_{2}\right)-1} u_{2}(\mathbf{x}), 0\right) \cdot \mathbf{u} d \mathbf{x} \\
I_{3}:=-\int_{\Omega}(\mathbf{u} \cdot \nabla) \mathbf{u}_{2} \cdot \mathbf{u} d \mathbf{x} .
\end{gathered}
$$

Estimate for the temperature. Using (4.35) and Cauchy's inequality, we get

$$
\left|J_{1}\right| \leq \lambda \int_{\Omega}|\theta|\left|\mathbf{u}_{1}\right||\nabla \theta| d \mathbf{x} \leq \frac{1}{4} \int_{\Omega}|\nabla \theta|^{2} d \mathbf{x}+\lambda^{2} \int_{\Omega}|\theta|^{2}\left|\mathbf{u}_{1}\right|^{2} d \mathbf{x}
$$

and

$$
\left|J_{2}\right| \leq C \int_{\Omega}|\mathbf{u}||\nabla \theta| d \mathbf{x} \leq \frac{1}{4} \int_{\Omega}|\nabla \theta|^{2} d \mathbf{x}+C^{2} \int_{\Omega}|\mathbf{u}|^{2} d \mathbf{x},
$$

with $C=C(m, M)=\max _{m \leq \theta \leq M}|\mathcal{C}(\theta)|$. In the sequel the letter $C$ will be used for different constants depending on $L, m, M, \delta$ and $\nu$. We use the Poincaré inequalities

$$
\int_{0}^{L}|\mathbf{u}|^{2} d y \leq C \int_{\Omega}|\nabla \mathbf{u}|^{2} d \mathbf{x}
$$

and

$$
|\theta(x, y)|^{2} \leq L \int_{0}^{L}\left|\theta_{y}(x, s)\right|^{2} d s
$$

to obtain, from (3.31), that

$$
\begin{gathered}
\int_{\Omega}|\theta|^{2}\left|\mathbf{u}_{1}\right|^{2} d \mathbf{x} \leq \\
C \int_{0}^{\infty}\left(\int_{0}^{L}\left|\theta_{y}(x, s)\right|^{2} d s\right)\left(\int_{\Omega}\left|\nabla \mathbf{u}_{1}\right|^{2} d \mathbf{x}\right) d x \leq C \int_{\Omega}|\nabla \theta|^{2} d \mathbf{x} .
\end{gathered}
$$

Joining (4.37), (4.39), (4.40) and (4.43), we arrive to

$$
\frac{1}{2} \int_{\Omega}|\nabla \theta|^{2} d \mathbf{x} \leq C \lambda^{2} \int_{\Omega}|\nabla \theta|^{2} d \mathbf{x}+C \int_{\Omega}|\nabla \mathbf{u}|^{2} d \mathbf{x} .
$$

Choosing $\lambda$ such that

$$
2 C \lambda^{2}<1
$$

it results

$$
\int_{\Omega}|\nabla \theta|^{2} d \mathbf{x} \leq C \int_{\Omega}|\nabla \mathbf{u}|^{2} d \mathbf{x}
$$

Estimate for the velocity. Applying the inequality

$$
\sigma|\xi-\eta|^{\sigma+1} \leq\left(|\xi|^{\sigma-1} \xi-|\eta|^{\sigma-1} \eta\right)(\xi-\eta)\left(|\xi|^{\sigma+1}+|\eta|^{\sigma+1}\right)^{\frac{1-\sigma}{1+\sigma}}
$$


with $0<\sigma<1$ and using (1.2), we can write

$$
\begin{gathered}
0<\delta \sigma^{-} \int_{\Omega} \chi_{\mathbf{f}}(\mathbf{x})\left|u_{1}-u_{2}\right|^{\sigma\left(\theta_{1}\right)+1}\left(\left|u_{1}\right|^{\sigma\left(\theta_{1}\right)+1}+\left|u_{2}\right|^{\sigma\left(\theta_{1}\right)+1}\right)^{\frac{\sigma\left(\theta_{1}\right)-1}{\sigma\left(\theta_{1}\right)+1}} d \mathbf{x} \\
\leq I_{1} .
\end{gathered}
$$

By Lagrange's theorem,

$$
\left|u_{2}\right|^{\sigma\left(\theta_{1}\right)-1} u_{2}-\left|u_{2}\right|^{\sigma\left(\theta_{2}\right)-1} u_{2}=\sigma^{\prime}\left(\theta_{*}\right)\left|u_{2}\right|^{\sigma\left(\theta_{*}\right)} \ln \left|u_{2}\right| \theta
$$

for every $\theta_{*}$ in the interval with extremities $\theta_{1}$ and $\theta_{2}$. Then we conclude

$$
\left|I_{2}\right| \leq \delta \int_{\Omega}\left|\sigma^{\prime}\right|\left|u_{2}\right|^{\sigma\left(\theta_{*}\right)} \ln \left|u_{2}\right||\theta||\mathbf{u}| d \mathbf{x}
$$

By (4.36), Cauchy's inequality and (4.41) we obtain

$$
\left|I_{2}\right| \leq \frac{\nu}{2} \int_{\Omega}|\nabla \mathbf{u}|^{2} d \mathbf{x}+\lambda^{2} C I_{21}, I_{21}=\int_{\Omega}|\theta|^{2}\left|u_{2}\right|^{2 \sigma\left(\theta_{*}\right)}\left(\ln \left|u_{2}\right|\right)^{2} d \mathbf{x} .
$$

Using (4.42) we get

$$
I_{21} \leq L \int_{0}^{\infty}\left(\int_{0}^{L}|\nabla \theta(x, s)|^{2} d s\right)\left(\int_{0}^{L}\left|u_{2}(x, y)\right|^{2 \sigma\left(\theta_{*}\right)}\left(\ln \left|u_{2}\right|\right)^{2} d y\right) d x .
$$

Now we recall the following elementary inequalities

$$
\begin{gathered}
\left|u_{2}\right|^{2 \sigma\left(\theta_{*}\right)}\left(\ln \left|u_{2}\right|\right)^{2} \leq C \text { for }\left|u_{2}\right| \leq 1, \quad C=C\left(\sigma^{-}, \sigma^{+}\right) \\
\left|u_{2}\right|^{2 \sigma\left(\theta_{*}\right)}\left(\ln \left|u_{2}\right|\right)^{2} \leq \frac{1}{\varepsilon^{2}}\left|u_{2}\right|^{2} \quad \text { for }\left|u_{2}\right| \geq 1, \varepsilon=1-\sigma^{+}>0 .
\end{gathered}
$$

Then, separating in two integrals for $\left|u_{2}\right|<1$ and for $\left|u_{2}\right| \geq 1$, we obtain

$$
\int_{0}^{L}\left|u_{2}(x, y)\right|^{2 \sigma\left(\theta_{*}\right)}\left(\ln \left|u_{2}\right|\right)^{2} d y \leq C\left(1+\int_{0}^{L}\left|u_{2}(x, y)\right|^{2} d y\right) .
$$

Using (3.31) and (4.41)

$$
\int_{0}^{L}\left|u_{2}(x, y)\right|^{2 \sigma\left(\theta_{*}\right)}\left(\ln \left|u_{2}\right|\right)^{2} d y \leq C .
$$

Finally we obtain

$$
I_{21} \leq C \int_{\Omega}|\nabla \theta|^{2} d \mathbf{x}
$$

and consequently

$$
\left|I_{2}\right| \leq \frac{\nu}{2} \int_{\Omega}|\nabla \mathbf{u}|^{2} d \mathbf{x}+\lambda^{2} C \int_{\Omega}|\nabla \theta|^{2} d \mathbf{x}
$$

Using (4.45)

$$
\left|I_{2}\right| \leq \frac{\nu}{2} \int_{\Omega}|\nabla \mathbf{u}|^{2} d \mathbf{x}+\lambda^{2} C \int_{\Omega}|\nabla \mathbf{u}|^{2} d \mathbf{x} .
$$


Last inequality, (4.38) and (4.46), give us

$$
\begin{gathered}
\nu \int_{\Omega}|\nabla \mathbf{u}|^{2} d \mathbf{x}+\delta \int_{\Omega} \chi_{\mathbf{f}}(\mathbf{x})\left|u_{1}-u_{2}\right|^{\sigma\left(\theta_{1}\right)-1}\left(\left|u_{1}\right|^{\sigma\left(\theta_{1}\right)+1}+\left|u_{2}\right|^{\sigma\left(\theta_{1}\right)+1}\right)^{\frac{\sigma\left(\theta_{1}\right)-1}{\sigma\left(\theta_{1}\right)+1}} d \mathbf{x} \\
\leq\left|I_{3}\right|+\left(\frac{\nu}{2}+\lambda^{2} C\right) \int_{\Omega}|\nabla \mathbf{u}|^{2} d \mathbf{x}
\end{gathered}
$$

Choosing $\lambda$ in (4.47) such that

$$
2 C \lambda^{2}<\nu
$$

we get that

$$
\begin{gathered}
\int_{\Omega}|\nabla \mathbf{u}|^{2} d \mathbf{x}+\delta \int_{\Omega} \chi_{\mathbf{f}}(\mathbf{x})\left|u_{1}-u_{2}\right|^{\sigma\left(\theta_{1}\right)-1}\left(\left|u_{1}\right|^{\sigma\left(\theta_{1}\right)+1}+\left|u_{2}\right|^{\sigma\left(\theta_{1}\right)+1}\right)^{\frac{\sigma\left(\theta_{1}\right)-1}{\sigma\left(\theta_{1}\right)+1}} d \mathbf{x} \\
\leq C\left|I_{3}\right| .
\end{gathered}
$$

By using (3.31) and some well-known estimates (see, e.g., [9, 12]) we can estimate $\left|I_{3}\right|$ in the following way

$$
\begin{gathered}
\left|I_{3}\right| \leq \int_{\Omega}\left|\nabla \mathbf{u}_{2}\right||\mathbf{u}|^{2} d \mathbf{x} \\
\leq\left\|\nabla \mathbf{u}_{\mathbf{2}}\right\|_{\mathbf{L}^{2}(\Omega)}\|\mathbf{u}\|_{\mathbf{L}^{4}(\Omega)}^{2} \leq C\left\|\mathbf{u}_{*}\right\|_{\mathbf{H}^{\frac{1}{2}(0, L)}}\|\nabla \mathbf{u}\|_{\mathbf{L}^{2}(\Omega)}^{2} .
\end{gathered}
$$

Thus, by assuming that $C\left\|\mathbf{u}_{*}\right\|_{\mathbf{H}^{\frac{1}{2}(0, L)}}<1$, using (4.49) and Poincaré's inequality (3.19), we obtain, from (4.48), that $\mathbf{u}_{1}=\mathbf{u}_{2}$ and, as consequence of (4.45), $\theta_{1}=\theta_{2}$.

Remark 4.1. The conditions (4.35) and (4.36) may be replaced by the condition

$$
M-m=\lambda
$$

for some $\lambda$ small enough, where $m$ and $M$ are given in (2.15). Here $\sigma, \mathcal{C} \in$ $\mathrm{C}^{2}(m, M)$ and according to Lagrange's theorem, $\mathcal{C}^{\prime}=\mathcal{C}^{\prime \prime} \theta$ and $\sigma^{\prime}=\sigma^{\prime \prime} \theta$. Then $\left|\mathcal{C}^{\prime}\right| \leq \max _{\theta \in[m, M]}\left|\mathcal{C}^{\prime \prime}\right||M-m| \leq C \lambda$ and $\left|\sigma^{\prime}\right| \leq \max _{\theta \in[m, M]}\left|\sigma^{\prime \prime}\right||M-m| \leq C \lambda$.

Remark 4.2. It seems possible to prove the uniqueness of solutions for the problem (2.7)-(2.14) with the body forces field given by (3.22) by proceeding as in [4] once we assume the following non-increasing condition

$$
\left(\mathbf{f}\left(\mathbf{x}, \theta, \mathbf{u}_{1}\right)-\mathbf{f}\left(\mathbf{x}, \theta, \mathbf{u}_{2}\right)\right) \cdot\left(\mathbf{u}_{1}-\mathbf{u}_{2}\right) \leq 0
$$

for every $\mathbf{u}_{1}, \mathbf{u}_{2} \in \mathbb{R}^{2}$, for any $\theta \in[m, M]$ and almost all $\mathbf{x} \in \Omega$. 


\section{Localization effect}

In this section we study the localization effect for the velocity $\mathbf{u}$ associated to the problem (2.7)-(2.14). It turns out that the qualitative property of the spatial localization of $\mathbf{u}$ is independent of the temperature component $\theta$. So, if we are not interested to know how big is the support of $\mathbf{u}$ but merely in knowing that support of $\mathbf{u}$ is a compact subset of $\Omega$ we can assume $\theta$ be given. In this way, our problem becomes simpler than before (since there is none $\mathrm{PDE}$ for $\theta$ ) and so, given $\theta$ such that

$$
\theta \in \mathrm{L}^{\infty}(\Omega), \quad \theta(\mathbf{x}) \in[m, M] \text { for a.e. } \mathbf{x} \in \Omega .
$$

we consider the following auxiliary problem

$$
\begin{gathered}
(\mathbf{u} \cdot \nabla) \mathbf{u}=\nu \triangle \mathbf{u}-\nabla p+\mathbf{f}(\mathbf{x}, \theta, \mathbf{u}), \\
\operatorname{div} \mathbf{u}=0, \\
\mathbf{u}=\mathbf{u}_{*}, \quad \text { on } x=0, \\
\mathbf{u}=\mathbf{0}, \quad \text { on } y=0, L, \\
\mathbf{u} \rightarrow \mathbf{0}, \quad \text { when } x \rightarrow \infty,
\end{gathered}
$$

where the forces field satisfy (1.1)-(1.3). In Section 3 (see (3.31)) has been established the existence of a weak solution $\mathbf{u}$ having a finite global energy

$$
E:=\int_{\Omega}\left(|\nabla \mathbf{u}|^{2}+\chi_{\mathbf{f}}|u|^{1+\sigma(\theta)}\right) d \mathbf{x}
$$

and consequently, from (1.2) and assuming that $|u| \leq 1$,

$$
\mathcal{E}:=\int_{\Omega}\left(|\nabla \mathbf{u}|^{2}+\chi_{\mathbf{f}}|u|^{1+\sigma^{+}}\right) d \mathbf{x}<\infty .
$$

As in $[3,4]$ we introduce the associated stream function $\psi$

$$
u=\psi_{y} \quad \text { and } \quad v=-\psi_{x} \quad \text { in } \Omega
$$

and we reduce the study of problem (5.52)-(5.56), to the consideration of the following fourth order problem where the pressure term does not appear anymore,

$$
\begin{gathered}
\nu \triangle^{2} \psi+\frac{\partial f_{1}}{\partial y}-\frac{\partial f_{2}}{\partial x}=\psi_{y} \triangle \psi_{x}-\psi_{x} \triangle \psi_{y} \quad \text { in } \Omega, \\
\psi(x, 0)=\psi(x, L)=\frac{\partial \psi}{\partial n}(x, 0)=\frac{\partial \psi}{\partial n}(x, L)=0 \quad \text { for } x \in(0, \infty), \\
\psi(0, y)=\int_{0}^{y} u_{*}(s) d s, \frac{\partial \psi}{\partial n}(0, y)=v_{*}(y) \quad \text { for } y \in(0, L), \\
\psi(x, y),|\nabla \psi(x, y)| \rightarrow 0, \text { as } x \rightarrow \infty \quad \text { and for } y \in(0, L) .
\end{gathered}
$$

Here $\mathbf{f}=\left(f_{1}, f_{2}\right)=\left(f_{1}\left(\mathbf{x}, \theta, \psi_{y},-\psi_{x}\right), f_{2}\left(\mathbf{x}, \theta, \psi_{y},-\psi_{x}\right)\right)$ and we recall that $\theta$ is assumed to be given. The notion of weak solution is adapted again to the information we have on the function $\mathbf{f}$. 
Definition 5.1. Given $\theta$ satisfying (5.51), a function $\psi$ is a weak solution of problem (5.60)-(5.63), if:

(i) $\psi \in \mathrm{H}^{2}(\Omega), \mathbf{f}\left(\mathbf{x}, \theta, \psi_{y},-\psi_{x}\right) \in \mathbf{L}_{\mathrm{loc}}^{1}(\Omega)$;

(ii) $\psi(0, y)=\int_{0}^{y} u_{*}(s) d s, \frac{\partial \psi}{\partial n}(0, y)=v_{*}(y), \psi(x, 0)=\psi(x, L)=\frac{\partial \psi}{\partial n}(x, 0)=$ $\frac{\partial \psi}{\partial n}(x, L)=\psi(0, L)=0$, and $\psi,|\nabla \psi| \rightarrow 0$, when $x \rightarrow \infty ;$

(iii) For every $\phi \in \mathrm{H}_{0}^{2}(\Omega) \cap \mathrm{W}^{1, \infty}(\Omega)$ with compact support,

$\nu \int_{\Omega} \triangle \psi \triangle \phi d \mathbf{x}-\int_{\Omega}\left(f_{1} \phi_{y}-f_{2} \phi_{x}\right) d \mathbf{x}=\int_{\Omega} \triangle \psi\left(\psi_{x} \phi_{y}-\psi_{y} \phi_{x}\right) d \mathbf{x}$.

To establish the localization effect, we proceed as in $[3,4]$ and we prove the followings lemmas.

Lemma 5.1. Given $\theta$ satisfying (5.51), if $\mathbf{u}$ is a weak solution of $\{(5.52)-(5.56)$, (1.1) $-(1.3)\}$ in the sense of (ii) of Definition 3.1, then $\psi$, given by (5.59), is a weak solution of (5.60)-(5.63) in the sense of Definition 5.1 .

Lemma 5.2. Given $\theta$ satisfying (5.51), let $\psi$ be a weak solution of (5.60)-(5.63) with $E$ finite. Assume that $\mathbf{f}$ satisfies (1.1)-(1.3) with $x_{\mathbf{f}}=\infty$. Then, for every $a>x_{g}$, and every positive integer $m \geq 2$

$$
\begin{gathered}
\int_{\Omega}\left(\nu\left|\mathrm{D}^{2} \psi\right|^{2}+\delta\left|\psi_{y}\right|^{1+\sigma^{+}}\right)(x-a)_{+}^{m} d \mathbf{x} \\
\leq 2 m \nu \int_{\Omega}|\triangle \psi|\left|\psi_{x}\right|(x-a)_{+}^{m-1} d \mathbf{x}+2 m \nu \int_{\Omega}\left|\psi_{y}\right|\left|\psi_{x y}\right|(x-a)_{+}^{m-1} d \mathbf{x} \\
+m(m-1) \nu \int_{\Omega}|\triangle \psi||\psi|(x-a)_{+}^{m-2} d \mathbf{x}+m \int_{\Omega}|\triangle \psi|\left|\psi_{y}\right||\psi|(x-a)_{+}^{m-1} d \mathbf{x}
\end{gathered}
$$

where $\left|\mathrm{D}^{2} \psi\right|^{2}=\psi_{x x}^{2}+2 \psi_{x y}^{2}+\psi_{y y}^{2}$.

From the left-hand side of (5.65), it will arise the energy type term which depends on $a$

$$
\mathcal{E}_{m}(a)=\int_{\Omega}\left(\left|\mathrm{D}^{2} \psi\right|^{2}+\left|\psi_{y}\right|^{1+\sigma^{+}}\right)(x-a)_{+}^{m} d \mathbf{x}
$$

and we observe that

$$
\mathcal{E}_{0}(0)=\mathcal{E}, \quad\left(\mathcal{E}_{m}(a)\right)^{(k)}=(-1)^{k} \frac{m !}{(m-k) !} \mathcal{E}_{m-k}(a), 0 \leq k \leq m .
$$

Then, the following lemma is proved as in [4], where now $\sigma$ depends on the temperature $\theta$ and satisfies (1.2).

Lemma 5.3. Let $\psi$ be a weak solution of (5.60)-(5.63) and let us assume $\mathbf{f}$ satisfies (1.1)-(1.3) with $x_{\mathbf{f}}=\infty$. Then, the following differential inequality holds for $a \geq x_{g}$ $\left(x_{g}\right.$ is given in $\left.(1.3)\right)$ :

$$
\mathcal{E}_{m}(a) \leq C\left(\mathcal{E}_{m-2}(a)\right)^{\mu_{1}}+C\left(\mathcal{E}_{m-2}(a)\right)^{\mu_{2}}, \quad \text { for any } \theta \in[m, M]
$$


for every integer $m>3$, where $C=C\left(L, m, \delta, \nu, \sigma^{ \pm}\right)$are different positive constants and $\mu_{j}=\mu_{j}\left(m, \sigma^{+}\right)>1, j=1,2$. Moreover, $\mathcal{E}_{2}(a)<\infty$ for any $a \geq x_{g}$. In fact,

$$
\mathcal{E}_{2}(a) \leq C\left(\mathcal{E}_{0}(a)\right)^{\mu_{1}}+C\left(\mathcal{E}_{0}(a)\right)^{\mu_{2}}, \quad \text { for any } \theta \in[m, M]
$$

where $C$ are different positive constants, the first an absolute constant and the others such that $C=C\left(L, \delta, \nu, \sigma^{ \pm}\right)$, and $\mu_{j}=\mu_{j}\left(\sigma^{+}\right)>1, j=1,2$.

Starting with the case $x_{\mathbf{f}}=\infty$, we take $m=4$ in Lemma 5.3 and then we have the fractional differential inequality

$$
\mathcal{E}_{4}(a) \leq C\left(\mathcal{E}_{2}(a)\right)^{\mu_{1}}+C\left(\mathcal{E}_{2}(a)\right)^{\mu_{2}},
$$

where, according to what we have done in [4], $\mu_{j}=\mu_{j}\left(\sigma^{+}\right)>1, j=1,2$ and $C=C\left(L, m, \delta, \nu, \sigma^{ \pm}\right)$means two different positive constants. Using Lemma 5.3 with $m=2$ and because of the finiteness of $\mathcal{E}$ (see (5.58)), we can easily see that $\mathcal{E}_{2}(a)$ is finite. Then, using Lemma 5.1 of [4] and proceeding as in this last reference, we prove the support of $\mathcal{E}_{0}(a)$ is a bounded interval $\left[0, a^{*}\right]$ with $a^{*} \leq a^{\prime}$, where $a^{\prime}$ is an upper limit to $a^{*}$ and given by

$$
a^{\prime}=\frac{C}{1-\sigma^{+}} \mathcal{E}^{\frac{1}{2\left(7+\sigma^{+}\right)}}, \quad C=C\left(E, L, \delta, \nu, \sigma^{ \pm}\right) .
$$

Then $\mathcal{E}_{0}(a)=0$ for $a>a^{\prime}$, which implies $\mathbf{u}=\mathbf{0}$ almost everywhere for $x>a^{\prime}$.

For the case $x_{\mathbf{f}}<\infty$, the proof follows exactly as in [3].

Remark 5.1. We obtain the same localization effect if we consider the non-constant semi-infinite strip $\Omega=(0, \infty) \times\left(L_{1}(x), L_{2}(x)\right)$, with $L_{1}, L_{2} \in \mathrm{C}^{2}(0, \infty), k_{1} \leq$ $\left|L_{2}(x)-L_{1}(x)\right| \leq k_{2},\left|L_{1}^{\prime}(x)\right|,\left|L_{2}^{\prime}(x)\right| \leq k_{3}$, and $\left|L_{1}^{\prime \prime}(x)\right|,\left|L_{2}^{\prime \prime}(x)\right| \leq k_{4}$ for all $x \geq 0$, where $k_{i}, i=1, \ldots, 4$, are positive constants.

\section{Case of a temperature depending viscosity}

A harder, but very interesting, problem arises when the viscosity depends also on the temperature (which is very often the case in many concrete applications). In this case, the equation of motion (5.52) must be replaced by

$$
(\mathbf{u} \cdot \nabla) \mathbf{u}=\operatorname{div}(2 \nu(\theta) \mathbf{D})-\nabla p+\mathbf{f}(\mathbf{x}, \theta, \mathbf{u}),
$$

where $\mathbf{D}=\left(\nabla \mathbf{u}+\nabla \mathbf{u}^{T}\right) / 2$ is the rate of strain tensor. We assume that

$$
0<\nu^{-} \leq \nu(\theta) \leq \nu^{+}<\infty,
$$

for some constants $\nu^{-}$and $\nu^{+}$, and the equation (3.21) of (ii) of Definition 3.1 is replaced by

$$
2 \int_{\Omega} \nu(\theta) \mathbf{D}: \nabla \varphi d \mathbf{x}+\int_{\Omega} \mathbf{u} \cdot \nabla \mathbf{u} \cdot \varphi d \mathbf{x}=\int_{\Omega} \mathbf{f} \cdot \varphi d \mathbf{x} .
$$

The main goal of this section is to indicate how the localization effect can be proved still in this case. We assume the existence of, at least, one weak solution 
$(\theta, \mathbf{u})$, in the sense of Definition 3.1 with (3.21) replaced by (6.68), to problem (6.66), (2.5)-(2.14) having a finite global energy (5.57).

To establish the localization effect, we proceed as in Section 5 by introducing the stream function (5.59) associated with the vector velocity and we reduce the problem $\{(6.66),(5.53)-(5.56)\}$ to the following one,

$$
\begin{gathered}
{\left[\nu(\theta)\left(\psi_{x x}-\psi_{y y}\right)\right]_{x x}+\left[\nu(\theta)\left(\psi_{y y}-\psi_{x x}\right)\right]_{y y}+4\left[\nu(\theta) \psi_{x y}\right]_{x y}} \\
+\frac{\partial f_{1}}{\partial y}-\frac{\partial f_{2}}{\partial x}=\psi_{y} \triangle \psi_{x}-\psi_{x} \triangle \psi_{y} \\
\psi(x, 0)=\psi(x, L)=\frac{\partial \psi}{\partial n}(x, 0)=\frac{\partial \psi}{\partial n}(x, L)=0 \quad \text { for } x \in(0, \infty), \\
\psi(0, y)=\int_{0}^{y} u_{*}(s) d s, \frac{\partial \psi}{\partial n}(0, y)=v_{*}(y) \quad \text { for } y \in(0, L), \\
\psi(x, y),|\nabla \psi(x, y)| \rightarrow 0, \text { as } x \rightarrow \infty \quad \text { and for } y \in(0, L),
\end{gathered}
$$

where again $\mathbf{f}=\left(f_{1}, f_{2}\right)=\left(f_{1}\left(\mathbf{x}, \theta, \psi_{y},-\psi_{x}\right), f_{2}\left(\mathbf{x}, \theta, \psi_{y},-\psi_{x}\right)\right)$ and the notion of weak solution to problem (6.69)-(6.72) is adapted, from Definition 5.1, by replacing (5.64) by

$$
\begin{gathered}
\int_{\Omega} \nu(\theta)\left[\left(\psi_{x x}-\psi_{y y}\right)\left(\phi_{x x}-\phi_{y y}\right)+4 \psi_{x y} \phi_{x y}\right] d \mathbf{x} \\
-\int_{\Omega}\left(f_{1} \phi_{y}-f_{2} \phi_{x}\right) d \mathbf{x}=\int_{\Omega} \triangle \psi\left(\psi_{x} \phi_{y}-\psi_{y} \phi_{x}\right) d \mathbf{x} .
\end{gathered}
$$

In this case, the counterpart of (5.65) is

$$
\begin{gathered}
\int_{\Omega}\left(\nu^{-}\left|\mathrm{D}^{2} \psi\right|^{2}+\delta\left|\psi_{y}\right|^{1+\sigma^{+}}\right)(x-a)_{+}^{m} d \mathbf{x} \\
\leq 2 m \nu^{+} \int_{\Omega}\left(\left|\psi_{x x}\right|+\left|\psi_{y y}\right|\right)\left|\psi_{x}\right|(x-a)_{+}^{m-1} d \mathbf{x}+2 m \nu^{+} \int_{\Omega}\left|\psi_{x y}\right|\left|\psi_{y}\right|(x-a)_{+}^{m-1} d \mathbf{x} \\
+m(m-1) \nu^{+} \int_{\Omega}\left(\left|\psi_{x x}\right|+\left|\psi_{y y}\right|\right)|\psi|(x-a)_{+}^{m-2} d \mathbf{x} \\
+m \int_{\Omega}|\triangle \psi|\left|\psi_{y}\right||\psi|(x-a)_{+}^{m-1} d \mathbf{x} .
\end{gathered}
$$

Proceeding as in Section 5 and using the assumptions (1.2) and (6.67), we obtain the same localization effect mentioned in the precedent section.

\section{References}

[1] S.N. Antontsev, J.I. Díaz, H.B. de Oliveira. Stopping a viscous fluid by a feedback dissipative external field: I. The stationary Stokes equations. Book of abstracts of NSEC8, Euler International Mathematical Institute, St. Petersburg, 2002.

[2] S.N. Antontsev, J.I. Díaz, H.B. de Oliveira. On the confinement of a viscous fluid by means of a feedback external field. C.R. Mécanique 330 (2002), 797-802.

[3] S.N. Antontsev, J.I. Díaz, H.B. de Oliveira. Stopping a viscous fluid by a feedback dissipative field: I. The stationary Stokes problem. To appear in J. Math. Fluid Mech. 
[4] S.N. Antontsev, J.I. Díaz, H.B. de Oliveira. Stopping a viscous fluid by a feedback dissipative field: I. The stationary Navier-Stokes problem. To appear in Rend. Lincei Mat. Appl.

[5] S.N. Antontsev, J.I. Díaz, S.I. Shmarev. Energy Methods for Free Boundary Problems: Applications to Non-linear PDEs and Fluid Mechanics. Progress in Nonlinear Differential Equations and Their Applications, Vol. 48, Birkhäuser, Boston, 2002.

[6] J.R. Canon, E. DiBenedetto, G.H. Knightly. The bidimensional Stefan problem with convection: the time-dependent case. Comm. Partial Differential Equations, 8 (1983), 1549-1604.

[7] J. Carrillo, M. Chipot, On some nonlinear elliptic equations involving derivatives of the nonlinearity, Proc. Roy. Soc. Edinburgh Sect. A 100 (3-4) (1985), 281-294.

[8] E. DiBenedetto, M. O'Leary. Three-dimensional conduction-convection problems with change of phase. Arch. Rational Mech. Anal. 123 (1993), 99-117.

[9] G.P. Galdi. An Introduction to the Mathematical Theory of the Navier-Stokes Equations: Nonlinear Steady Problems. Springer-Verlag, New York, 1994.

[10] D. Gilbarg, N.S. Trudinger. Elliptic Partial Differential Equations of Second Order. Springer-Verlag, Berlin Heidelberg, 1998.

[11] O.A. Ladyzhenskaya, N.N. Ural'tseva. Linear and Quasilinear Elliptic Equations. Academic Press, New York, 1968.

[12] O.A. Ladyzhenskaya. The Mathematical Theory of Viscous Incompressible Fluids. Gordon and Breach Science Publishers Inc., New York, 1969.

[13] V.A. Solonnikov. On the solvability of boundary and initial boundary value problems for the Navier-Stokes systems in domains with noncompact boundaries. Pacific J. Math. 93 (2) (1981), 443-458.

[14] X. Xu, M. Shillor. The Stefan problem with convection and Joule's heating. Adv. Differential Equations 2 (1997), 667-691.

\author{
S.N. Antontsev \\ Universidade da Beira Interior \\ Covilhã, Portugal \\ e-mail: anton@ubi.pt \\ J.I. Díaz \\ Universidad Complutense \\ Madrid, Spain \\ e-mail: ji_diaz@mat.ucm.es \\ H.B. de Oliveira \\ Universidade do Algarve \\ Faro, Portugal \\ e-mail: holivei@ualg.pt
}

